\title{
NUMERICAL ANALYSIS OF DYNAMICS OF AN AUTOMATICALLY TRACKED ANTI-TANK GUIDED MISSILE USING POLYNOMIAL FUNCTIONS
}

\author{
Zbigniew Koruba, Łukasz Nocoń \\ Kielce University of Technology, Faculty of Mechatronics and Machine Design, Kielce, Poland \\ e-mail:ksmzko@tu.kielce.pl,waldek.afro@op.pl
}

\begin{abstract}
The paper presents algorithms of automatic control of an intelligent anti-tank guided missile (ATGM) with possibility of attacking the target from the upper ceiling and with possibility of the missile flight through indicated points in space. The polynomial functions are used to designate the program trajectories. Numerical analysis of the operation of chosen algorithms is performed. The results are presented in a graphical form. As it results from the conducted tests, the proposed algorithms of automatic control of ATGM with the use of polynomial functions work properly during attack on the target from the upper ceiling, for both mobile and immobile targets.
\end{abstract}

Keywords: homing, direction algorithm, anti-tank guided missile

\section{Introduction}

This paper presents a medium-range anti-tank guided missile (ATGM) which is characterized by high maneuverability of flight, ability to attack a target from the upper hemisphere with the possibility to apply the feint. The passive guidance limits its tracking possibilities and the target can be selected from the battlefield during its flight.

High maneuverability of ATGM flight is directly used to bypass obstacles that appear on the flight trajectory of the missile. A special head is used to detect those obstacles. It is mounted on the deck of the ATGM, scanning the space in front of the ATGM (Nocon, 2013). Detecting an obstacle is done in real time. It allows for a suitable adjustment of the flight trajectory depending on the appearing obstacles. In the case of occurring terrain obstacles (natural such as hills, and artificial such as buildings), it is possible to determine permanent points in space which delineate a safe flight path of ATGM between those obstacles. Introducing a map of the battlefield area to the on-deck computer of the missile will allow the auto-pilot to autonomously delineate the optimum flight trajectory to the target. Moreover, thanks to the possibilities of determining the points of flight, the flight of the missile may be designed in such a way that it reaches the target along a circular trajectory, for example along a circle and, as a result, attack it from the least expected direction. During the programmed flight the missile realizes the trajectory set before the start with simultaneous consideration of bypassing the obstacles. In the last flight stage, the missile detects and identifies the target and fulfils the process of self-guidance in accordance with the implemented algorithm. Depending on the situation on the battlefield, two ways of guiding the missile can be selected. The first is self-guidance (the missile type "fire and forget"). It is fully automatic. After firing, the shooter has no control over the ATGM flight. The second way is "fire-observe-correct". In this case, the operator observes the actions on the battlefield in real time on the control panel. They operator can correct the flight path and choose targets. The image in real time is sent via a fiber-optic cable unwound behind the missile. 
Thanks to the use of the Observation and Seeker Head coupled with the combat head EFP, the missile can very accurately determine the location of the target and hit it in the least armoured place. In the paper, it is assumed that the ATGM is equipped with the modified scanning and tracking seeker which is presently undergoing intensive theoretical and experimental tests (Gapiński and Stefański, 2014; Krzysztofik, 2012).

During the attack, the flight of the missile is exposed to external disruptions in form of gusts of wind. Crosswinds surround the missile as a result of which the helm, flight stabilizers and the body of the missile are subject to additional, undesired carrying off forces and the moment of those forces. That results in flight trajectory errors. The auto-pilot with the proposed algorithm effectively compensates the occurring errors what was described in a separate paper by Nocoń and Stefański (2014). Even large gusts of wind of $17 \mathrm{~m} / \mathrm{s}$ do not impact significantly the effectiveness of the programmed flight, not to mention the attack itself. That is why external disruptions were not considered in flight dynamics equations.

This paper presents a much simplified physical model and flight dynamics equations which by no means affect the preliminary analysis of correctness and effectiveness of the control algorithm. It should be emphasized that the developed algorithm of controlling the hypothetical anti-tank guided missile is the essence of the paper. On the other hand, the equations of flight dynamics of the missile are the tool to verify the correctness of its operation. They have been derived and analysed in more detail in other papers (Koruba and Osiecki, 2006; Baranowski, 2013).

It seems that it is not an overstatement to claim that the missile described herein is a part of the most recent trend of the fourth generation of anti-tank missiles.

\section{Description of the tracking}

Contemporary military actions more and more often take place in urban areas with high density of civilian objects. With regard to civil security, the fact that the attack on hostile armored units should take place with simultaneous consideration of avoidance of all obstacles: buildings and civilian vehicles, allied units and permanent natural objects is important, see Fig. 1 (Koruba and Nocoń, 2012). Military actions are also common on wooded, mountainous and desert areas - there are also a lot of obstacles interfering with the trajectory of the flight of ATGM.

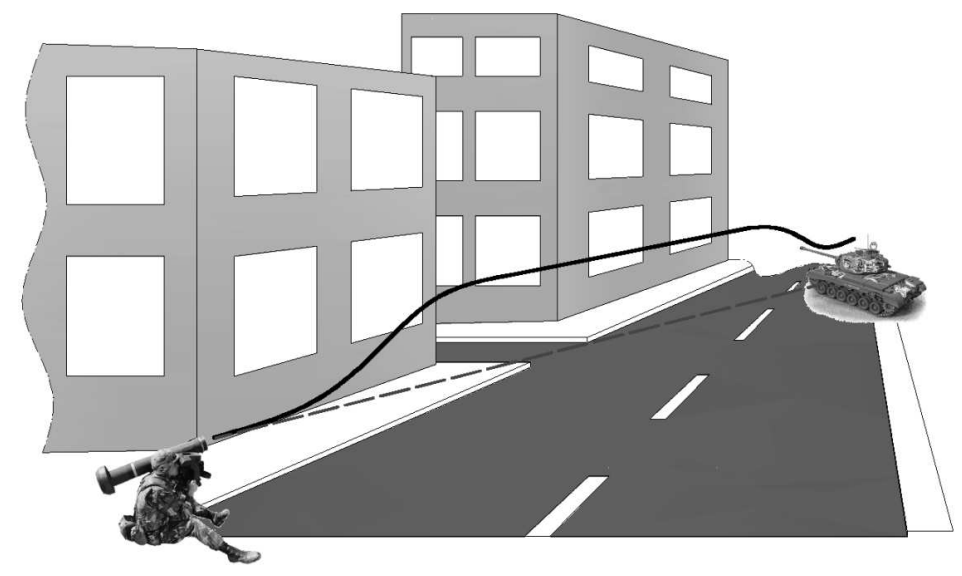

Fig. 1. General view of self-guidance of the third generation close-range ATGM in urban areas

The effectiveness of attack performance is influenced by the element of surprise, ability to avoid obstacles during the flight and the quality of armor of enemy's vehicles. It is commonly known that the weakest armor is the upper surface of the tank and its back, so it is best to direct the attack there. Based on the conditions presented above, the ATGM is required to enable efficient maneuvering between obstacles and precise hit on the selected target point. 
Given that the tactical withdrawal of tanks is done in reverse gear, it is possible to set the flight trajectory with a surprise maneuver, which involves flying around the tank and attacking it from behind.

\subsection{The modelling of ATGM motion}

The equations of dynamics of flight of an anti-tank guided missile are derived in accordance with the adopted assumption that the ATGM performs maneuvers mainly in the horizontal plane. The consequence is the order of rotations in the transformation the ground-fixed system $S x_{g} y_{g} z_{g}$ to the body-fixed system Sxyz (Koruba and Osiecki, 2006; Siouris, 2004). The first rotation is performed in accordance with the plane of change of the direction of the flight of the ATGM. The second is in accordance with the plane of change of altitude of flight of the ATGM.
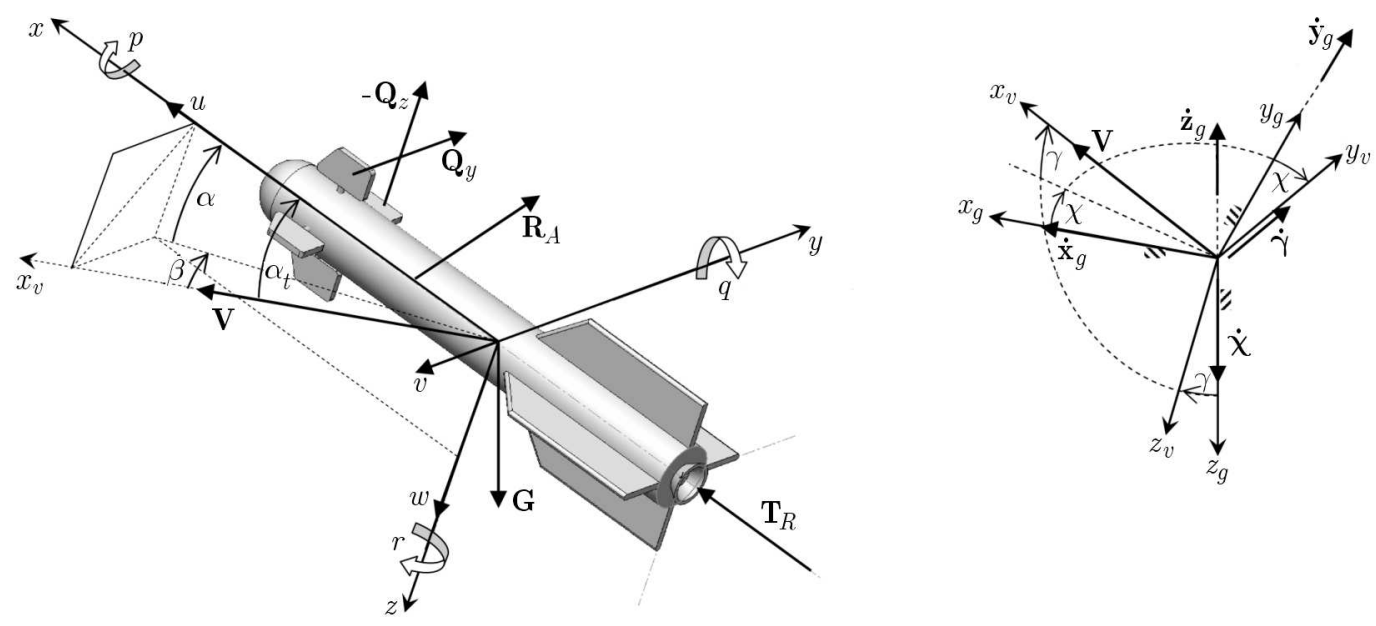

Fig. 2. The system of forces acting on the ATGM moving in the gravitational field and the Earth's atmosphere, together with the adopted coordinate systems

In Fig. 2, the following quantities and denotations are introduced: $\mathbf{R}_{A}$ - vector of total aerodynamic forces; $\mathbf{T}_{R}$ - total missile engine thrust; $\mathbf{G}$ - vector of the gravity force; $\mathbf{Q}_{y}, \mathbf{Q}_{z}-$ controlling forces; V - missile velocity vector; $S x y z$ - system of coordinates connected with the missile (body-fixed system); $S x_{g} y_{g} z_{g}$ - ground-fixed system; $S x_{v} y_{v} z_{v}$ - system of coordinates connected with the flow; $\alpha$ - missile angle of attack, $\alpha=\arctan (w / u) ; \beta$ - missile angle of sideslip, $\beta=\arcsin (v / V) ; \alpha_{t}$ - missile total angle of attack; $p, q, r$ - angular velocity components in the body reference frame; $u, v, w$ - velocity components in the body-fixed system; $\gamma, \chi$ - flight-path angle in the vertical and horizontal plane (inclination and azimuth angles of the missile velocity vector); $\dot{x}_{g}, \dot{y}_{g}, \dot{z}_{g}$ - components of the velocity vector in the ground-fixed system.

The dynamical equations of motion can be presented in different coordinate systems. In this paper, the mathematical model is developed according to Polish and International Standard ISO 1151. A transformation matrix between the ground-fixed system $S x_{g} y_{g} z_{g}$ and the bodyfixed system $S x y z$ is required to derive the equations of motion. The angular velocity is the sum of the rotation velocity with respect to the successive axes $\boldsymbol{\Omega}=\dot{\boldsymbol{\Theta}}+\dot{\boldsymbol{\Psi}}+\dot{\boldsymbol{\Phi}}$. The first rotation is around the vertical axis of the ground-fixed system $O z_{g}$ by the angle of azimuth $\Psi$, the second rotation is around the instantaneous horizontal axis $O y_{g}^{\prime}$ by the angle of inclination $\Theta$, and the third rotation is around the axis $O x$ by the angle of bank $\Phi$.

In Fig. 3, the following quantities $\mathbf{L}_{\Psi}, \mathbf{L}_{\Theta}$ and $\mathbf{L}_{\Phi}$ represent the transformation matrix: $\mathbf{L}_{\Psi}$ is the matrix of transformation of the rotation by the angle of azimuth $\Psi, \mathbf{L}_{\Theta}$ is the matrix of transformation of the rotation by the angle of inclination $\Theta, \mathbf{L}_{\Phi}$ is the matrix of transformation of the rotation by the angle of bank $\Phi$. 


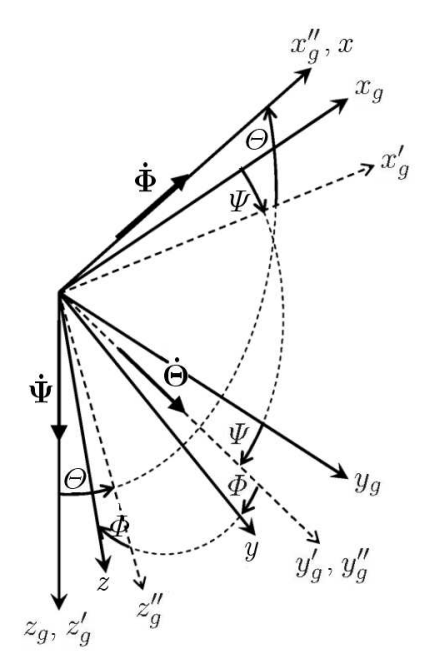

\begin{tabular}{c||c|c|c}
$L_{\Psi}$ & $x_{g}$ & $y_{g}$ & $z_{g}$ \\
\hline \hline$x_{g}^{\prime}$ & $\cos \Psi$ & $\sin \Psi$ & 0 \\
\hline$y_{g}^{\prime}$ & $-\sin \Psi$ & $\cos \Psi$ & 0 \\
\hline$z_{g}^{\prime}$ & 0 & 0 & 1
\end{tabular}

\begin{tabular}{c||c|c|c}
$L_{\Theta}$ & $x_{g}^{\prime}$ & $y_{g}^{\prime}$ & $z_{g}^{\prime}$ \\
\hline \hline$x_{g}^{\prime \prime}$ & $\cos \Theta$ & 0 & $-\sin \Theta$ \\
\hline$y_{g}^{\prime \prime}$ & 0 & 1 & 0 \\
\hline$z_{g}^{\prime \prime}$ & $\sin \Theta$ & 0 & $\cos \Theta$
\end{tabular}

\begin{tabular}{c||c|c|c}
$L_{\Phi}$ & $x_{g}^{\prime \prime}$ & $y_{g}^{\prime \prime}$ & $z_{g}^{\prime \prime}$ \\
\hline \hline$x$ & 1 & 0 & 0 \\
\hline$y$ & 0 & $\cos \Phi$ & $\sin \Phi$ \\
\hline$z$ & 0 & $-\sin \Phi$ & $\cos \Phi$
\end{tabular}

Fig. 3. Transformation the ground-fixed system $S x_{g} y_{g} z_{g}$ to the body-fixed system $S x y z$

The angular velocity components in the body-fixed system are calculated as follows

$$
\left[\begin{array}{c}
p \\
q \\
r
\end{array}\right]=\left[\begin{array}{c}
\dot{\Phi} \\
0 \\
0
\end{array}\right]+\mathbf{L}_{\Phi}\left[\begin{array}{c}
0 \\
\dot{\Theta} \\
0
\end{array}\right]+\mathbf{L}_{\Phi} \mathbf{L}_{\Theta}\left[\begin{array}{c}
0 \\
0 \\
\dot{\Psi}
\end{array}\right]
$$

The final form of the angular velocity is

$$
\begin{aligned}
& p=\dot{\Phi}-\dot{\Psi} \sin \Theta \quad q=\dot{\Theta} \cos \Phi+\dot{\Psi} \sin \Phi \cos \Theta \\
& r=-\dot{\Theta} \sin \Phi+\dot{\Psi} \cos \Phi \cos \Theta
\end{aligned}
$$

The dynamical equations of motion based on the principles of classical mechanics are divided into the progressive part of the missile motion and the spherical part of the motion. The first part of the dynamical equations is

$$
m \mathbf{a}=\sum \mathbf{F} \Rightarrow m \frac{\delta \mathbf{V}}{d t}=\sum \mathbf{F}
$$

The total velocity vector is the sum of the velocity components lying in the body-fixed system. Then, the derivative is computed

$$
\mathbf{V}=\mathbf{i} u+\mathbf{j} v+\mathbf{k} w \Rightarrow \frac{\delta \mathbf{V}}{d t}=\mathbf{i} \frac{\delta u}{d t}+\mathbf{j} \frac{\delta v}{d t}+\mathbf{k} \frac{\delta w}{d t}+u \frac{\delta \mathbf{i}}{d t}+v \frac{\delta \mathbf{j}}{d t}+w \frac{\delta \mathbf{k}}{d t}
$$

or

$$
\frac{\delta \mathbf{V}}{d t}=\mathbf{i} \dot{u}+\mathbf{j} \dot{v}+\mathbf{k} \dot{w}+\left|\begin{array}{ccc}
\mathbf{i} & \mathbf{j} & \mathbf{k} \\
p & q & r \\
u & v & w
\end{array}\right|
$$

The three dynamical equations of motion resulting from the progressive part of the motion are developed according with the body-fixed system

$$
m(\dot{u}+w q-v r)=\sum F_{x} \quad m(\dot{v}+u r-w p)=\sum F_{y} \quad m(\dot{w}+v p-u q)=\sum F_{z}
$$

The sum of the total forces acting on the missile is $\sum \mathbf{F}=\mathbf{T}_{R}+\mathbf{G}+\mathbf{R}_{A}+\mathbf{Q}_{S}$, where: $m$ is mass of the missile; $\mathbf{a}$ - total acceleration; $\mathbf{i}, \mathbf{j}, \mathbf{k}$ - unit vectors of the body-fixed system; $\mathbf{Q}_{S}=\left[0, Q_{y}, Q_{z}\right]$ - total vector of controlling forces. 
Based on the principle of angular momentum, the other part of dynamical equations is the spherical part of motion

$$
\frac{\delta \mathbf{K}}{d t}=\frac{\delta \mathbf{I} \Omega}{d t}=\sum \mathbf{M}
$$

The sum of the angular velocity in the body reference frame is the vector of the missile angular velocity $\boldsymbol{\Omega}=p \mathbf{i}+q \mathbf{j}+r \mathbf{k}$. The same case is with the angular momentum vector $\mathbf{K}=K_{x} \mathbf{i}+$ $K_{y} \mathbf{j}+K_{z} \mathbf{k}$. The derivative of the angular momentum vector is

$$
\frac{\delta \mathbf{K}}{d t}=\mathbf{i} \frac{\delta K_{x}}{d t}+\mathbf{j} \frac{\delta K_{y}}{d t}+\mathbf{k} \frac{\delta K_{z}}{d t}+K_{x} \frac{\delta \mathbf{i}}{d t}+K_{y} \frac{\delta \mathbf{j}}{d t}+K_{z} \frac{\delta \mathbf{k}}{d t}
$$

or

$$
\frac{\delta \mathbf{K}}{d t}=\mathbf{i} I_{x} \dot{p}+\mathbf{j} I_{y} \dot{q}+\mathbf{k} I_{z} \dot{r}+\left|\begin{array}{ccc}
\mathbf{i} & \mathbf{j} & \mathbf{k} \\
p & q & r \\
I_{x} p & I_{y} q & I_{z} r
\end{array}\right|
$$

The three dynamical equations of motion resulting from the spherical part of the motion are developed according with the body-fixed system

$$
\begin{array}{ll}
I_{x} \dot{p}+\left(I_{z}-I_{y}\right) q r=\sum M_{x} & I_{y} \dot{q}+\left(I_{x}-I_{z}\right) p r=\sum M_{y} \\
I_{z} \dot{r}+\left(I_{y}-I_{x}\right) p q & =\sum M_{z}
\end{array}
$$

The sum of the total moments acting on the missile is $\sum \mathbf{M}=\mathbf{M}_{A}+\mathbf{M}_{Q}$, where: $\mathbf{K}=\left[K_{x}, K_{y}, K_{z}\right]$ is the vector of the angular momentum components in the body-fixed system; $\boldsymbol{\Omega}$ - vector of the angular velocity; bf $I=\left[I_{x}, I_{y}, I_{z}\right]-$ moments of inertia; $\mathbf{M}_{A}=[L, M, N]-$ components of the total aerodynamic moment in the body-fixed system; $\mathbf{M}_{Q}$ - total moment of the controlling force.

- Forces and moments needed in the equations of motion

The total missile thrust $T_{R}$ is located in the axis $S x$ of the body-fixed system $S x y z$

$$
\left[\begin{array}{c}
T_{R}^{X} \\
T_{R}^{Y} \\
T_{R}^{Z}
\end{array}\right]=\left[\begin{array}{c}
T_{R} \\
0 \\
0
\end{array}\right]
$$

The vector of the gravity force $\mathbf{G}=\left[G_{x g}, G_{y g}, G_{z g}\right]$ is located in the axis $S z_{g}$ of the ground-fixed system $S x_{g} y_{g} z_{g}$, so $\mathbf{G}=[0,0, G]$ and thus it must be transformed to the body-fixed system Sxyz

$$
\left[\begin{array}{l}
G_{X} \\
G_{Y} \\
G_{Z}
\end{array}\right]=\mathbf{L}_{\Phi} \mathbf{L}_{\Theta} \mathbf{L}_{\Psi}\left[\begin{array}{l}
0 \\
0 \\
G
\end{array}\right]=\left[\begin{array}{c}
-G \sin \Theta \\
G \cos \Theta \sin \Phi \\
G \cos \Theta \cos \Phi
\end{array}\right]
$$

- Simplified aerodynamic forces and moments

The total aerodynamic force $\mathbf{R}_{A}$ is split into two components lying in plane of drag. The drag force $X_{A}=C_{D} \rho S V^{2} / 2$ is the component parallel to the vector of velocity, whereas the lift force $P_{A}=C_{L} \rho S V^{2} / 2$ is the component perpendicular to the vector of velocity.

The components of the total aerodynamic force $\mathbf{R}_{A}=[X, Y, Z]$ in the body-fixed system are

$$
\begin{aligned}
& X=-X_{A} \cos \alpha_{t}+P_{A} \sin \alpha_{t} \quad Y=\left(X_{A} \sin \alpha_{t}+P_{A} \cos \alpha_{t}\right) \cos \varphi \\
& Z=-\left(X_{A} \sin \alpha_{t}+P_{A} \cos \alpha_{t}\right) \sin \varphi
\end{aligned}
$$


(a)

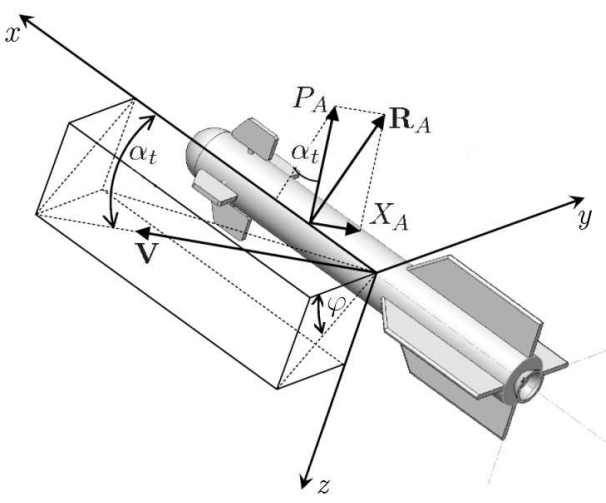

(b)

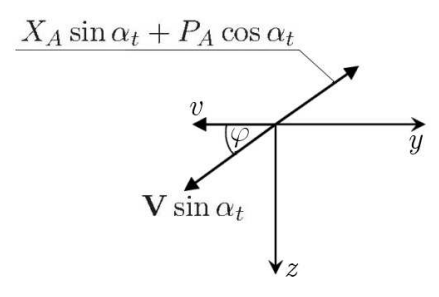

Fig. 4. The forces acting on the missile: (a) components and aerodynamic forces acting on the missile flight; (b) projections of the aerodynamic force vector $\mathbf{R}_{A}$ and the velocity vector $\mathbf{V}$ in the plane Syz

From obvious equations

$$
\begin{aligned}
& v=V \sin \alpha_{t} \cos \varphi \Rightarrow \cos \varphi=\frac{v}{V \sin \alpha_{t}} \\
& w=V \sin \alpha_{t} \sin \varphi \Rightarrow \sin \varphi=\frac{w}{V \sin \alpha_{t}}
\end{aligned}
$$

the final form of the components of the total aerodynamic force is

$$
\begin{aligned}
& X=-\frac{C_{D} \cos \alpha_{t}-C_{L} \sin \alpha_{t}}{2} \rho S V^{2} \quad Y=\frac{C_{D}+C_{L} \cot \alpha_{t}}{2} \rho S V v \\
& Z=-\frac{C_{D}+C_{L} \cot \alpha_{t}}{2} \rho S V w
\end{aligned}
$$

where: $C_{D}, C_{L}$ are coefficients of drag and lift; $\rho$ - air density; $S$ - cross sectional area of the missile; $\alpha_{t}=\arccos (u / V)$ - total angle of attack; $V=\sqrt{u^{2}+v^{2}+w^{2}}-$ missile velocity vector.

The vector of moments of the aerodynamic forces is $[L, M, N]=[0,-l Z,-l Y]$ in the body-fixed system, where $l$ is the distance from the center of gravity and the center of pressure of the aerodynamic forces.

The components of the total missile controlling forces $Q_{S}$ are located in the body-fixed system $S x y z$. In the axis $S y$ is the force of the directional control $Q_{y}$, in the axis $S z$ is the force of flight altitude control $Q_{z}$. The vector of moments of the controlling forces in the body-fixed system is $\mathbf{M}_{Q}=\left[0, e Q_{z},-e Q_{y}\right]$, where $e$ is the distance from the center of gravity and the control fins.

Based on a simplified physical model shown in Fig. 2 and on the assumption that the missile is axially symmetric $I_{y}=I_{z}$ and does not rotate around the longitudinal axis $S x$, equations (2.13) of the flight dynamics of the missile are derived. The dynamical equations consist of the progressive part of ATGM motion and its spherical part (Harris and Slegers, 2009; Koruba and Osiecki, 2006; Siouris, 2004)

$$
\begin{aligned}
& m(\dot{u}+w q-v r)=T_{R}-G \sin \Theta-\frac{C_{D} \cos \alpha_{t}-C_{L} \sin \alpha_{t}}{2} S \rho V^{2} \\
& m(\dot{v}+u r-w p)=G \cos \Theta \sin \Phi+\frac{C_{D}+C_{L} \cot \alpha_{t}}{2} S \rho V v+Q_{y} \\
& m(\dot{w}+v p-u q)=G \cos \Theta \cos \Phi-\frac{C_{D}+C_{L} \cot \alpha_{t}}{2} S \rho V w+Q_{z} \\
& I_{y} \dot{q}+\left(I_{x}-I_{z}\right) p r=-\frac{l}{2}\left(C_{D}+C_{L} \cot \alpha_{t}\right) \rho V w+e Q_{z} \\
& I_{z} \dot{r}+\left(I_{y}-I_{x}\right) p q=-\frac{l}{2}\left(C_{D}+C_{L} \cot \alpha_{t}\right) \rho V v-e Q_{y}
\end{aligned}
$$


The components of the velocity vector in the ground-fixed system (kinematic differential equations of motion of the missile center of mass) are as follows

$$
\left[\begin{array}{c}
\dot{x}_{g} \\
\dot{y}_{g} \\
\dot{z}_{g}
\end{array}\right]=\left(\mathbf{L}_{\Phi} \mathbf{L}_{\Theta} \mathbf{L}_{\Psi}\right)^{\mathrm{T}}\left[\begin{array}{c}
u \\
v \\
w
\end{array}\right]
$$

For the missile stabilized around the longitudinal axis $\Phi=0$, equation (2.14) takes the following form

$$
\begin{aligned}
& \dot{x}_{g}=u \cos \Theta \cos \Psi-v \sin \Psi+w \sin \Theta \cos \Psi \\
& \dot{y}_{g}=u \cos \Theta \sin \Psi+v \cos \Psi+w \sin \Theta \sin \Psi \\
& \dot{z}_{g}=-u \sin \Theta+w \cos \Theta
\end{aligned}
$$

The kinematic differential equations of rotational motion about the missile center of mass are as follows

$$
\begin{aligned}
& \dot{\Phi}=p+q \sin \Phi \tan \Theta+r \cos \Phi \tan \Theta \\
& \dot{\Theta}=q \cos \Phi-r \sin \Phi \quad \dot{\Psi}=q \frac{\sin \Phi}{\cos \Theta}+r \frac{\cos \Phi}{\cos \Theta}
\end{aligned}
$$

\subsection{Algorithm of control of the ATGM}

The control algorithm consists of two parts. The first part is a programmed trajectory that relates to the control in the vertical plane and the other part is a programmed trajectory in the horizontal plane. After combining both parts, we obtain the programmed trajectory of flight of the ATGM in space, running through the marked points (Fig. 5).

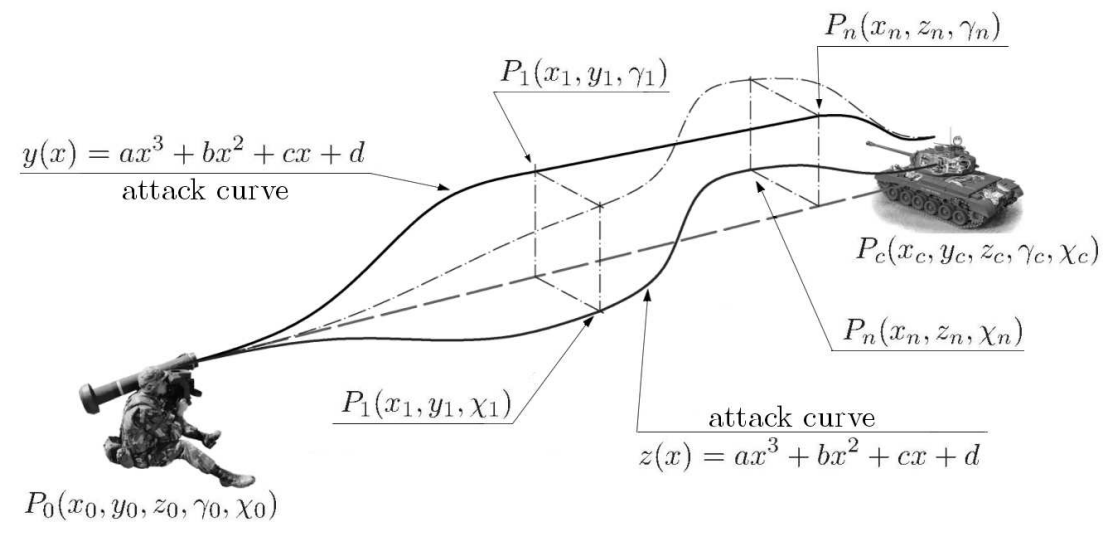

Fig. 5. A schematic depicting the gluing of the fragments of the trajectory by polynomial curves running through given points in space

A new fragment of both projections of the trajectory are calculated between the consecutive points. The program flight of the ATGM in each fragment is described by a third degree polynomial (Grzyb and Koruba, 2011)

$$
y=a x^{3}+b x^{2}+c x+d
$$

The program trajectory of flight of the ATGM consists of a finite number of sections - polynomial curves $y(x)$ and $z(x)$, glued with each other at predetermined points, see Fig. 5. Each curve is determined by the coordinates of the start and end point as well as angles of flight at these points. For the first section of the trajectory in the vertical plane, we can use the following data

$$
\left(x_{0}, y_{0}, \gamma_{0}\right)\left(x_{k}, y_{k}, \gamma_{k}\right)
$$


From the system of four equations with four unknowns, which are the coefficients with the variables

$$
\begin{array}{ll}
a x_{0}^{3}+b x_{0}^{2}+c x_{0}+d=y_{0} & 3 a x_{0}^{2}+2 b x_{0}+c=\tan \gamma_{0} \\
a x_{k}^{3}+b x_{k}^{2}+c x_{k}+d=y_{k} & 3 a x_{k}^{2}+2 b x_{k}+c=\tan \gamma_{k}
\end{array}
$$

we obtain the following linear equation to solve (Grzyb and Koruba, 2011)

$$
\left[\begin{array}{cccc}
x_{0}^{3} & x_{0}^{2} & x_{0} & 1 \\
3 x_{0}^{2} & 2 x_{0} & 1 & 0 \\
x_{k}^{3} & x_{k}^{2} & x_{k} & 1 \\
3 x_{k}^{2} & 2 x_{k} & 1 & 0
\end{array}\right]\left[\begin{array}{l}
a \\
b \\
c \\
d
\end{array}\right]=\left[\begin{array}{c}
y_{0} \\
\tan \gamma_{0} \\
y_{k} \\
\tan \gamma_{k}
\end{array}\right]
$$

In the attack phase (Fig. 6), when the target is moving, the polynomial coefficients are calculated at any point in time, as the end point (target) of the curve keeps changing its coordinates.

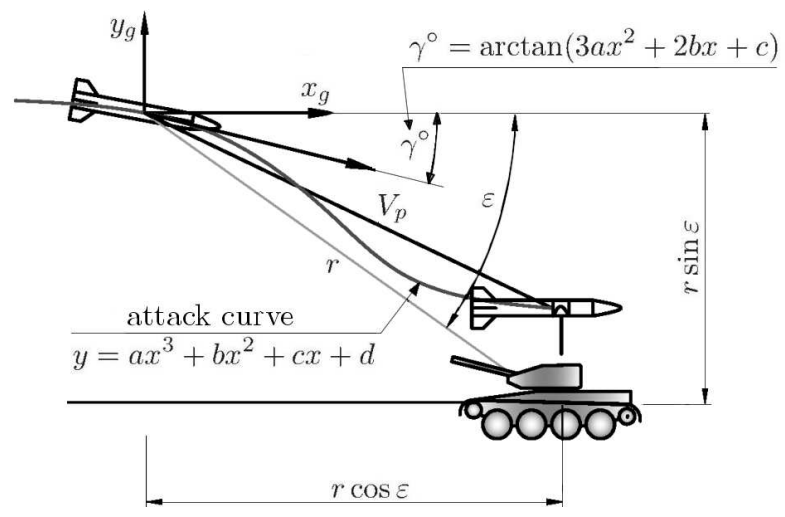

Fig. 6. View of the final stage of attack of the ATGM flying along a polynomial curve. The missile flight above the target

Due to the limited processing power of the system, the coefficients are presented in form of a cascade (next coefficient includes the previous one)

$$
\begin{array}{rlrl}
a & =-\frac{2 y_{k}-2 y_{0}+\left(x_{0}-x_{k}\right)\left(\tan \gamma_{k}+\tan \gamma_{0}\right)}{\left(x_{k}-x_{0}\right)^{3}} & c=\tan \gamma_{k}-3 x_{k}^{2} a-2 x_{k} b \\
b=\frac{\tan \gamma_{0}-\tan \gamma_{k}-\left(3 x_{0}^{2}-3 x_{k}^{2}\right) a}{2 x_{0}-2 x_{k}} & d=y_{k}-x_{k}^{3} a-x_{k}^{2} b-x_{k} c
\end{array}
$$

In the vertical plane, the program trajectory is described by the function $y=f(x)$

$$
y=a x^{3}+b x^{2}+c x+d
$$

The altitude control angle $\gamma^{\circ}$ is given by the formula

$$
\gamma^{\circ}=\arctan \left(3 a x^{2}+2 b x+c\right)
$$

The situation is analogous in the horizontal plane. By replacing the coordinate $y$ with $z$ and $\gamma$ with $\chi$, the program trajectory is described by the function $z=f(x)$

$$
z=a x^{3}+b x^{2}+c x+d
$$

and the direction control angle $\chi^{\circ}$

$$
\chi^{\circ}=\arctan \left(3 a x^{2}+2 b x+c\right)
$$




\subsection{Selection of a regulator}

To control the ATGM on the programmed trajectory in accordance with the implemented control algorithm, a double PID regulator is used in each plane separately

$$
\begin{aligned}
& Q_{y}=k_{y 1} e_{y}+k_{y 2} \frac{d e_{y}}{d t}+k_{y 3} \int_{t_{0}}^{t_{k}} e_{y} d t+h_{y 1} f_{y}+h_{y 2} \frac{d f_{y}}{d t}+h_{y 3} \int_{t_{0}}^{t_{k}} f_{y} d t \\
& Q_{z}=k_{z 1} e_{z}+k_{z 2} \frac{d e_{z}}{d t}+k_{z 3} \int_{t_{0}}^{t_{k}} e_{z} d t+h_{z 1} f_{z}+h_{z 2} \frac{d f_{z}}{d t}+h_{z 3} \int_{t_{0}}^{t_{k}} f_{z} d t
\end{aligned}
$$

where

$$
\begin{array}{ll}
e_{y}=\gamma^{\circ}-\gamma & e_{z}=\chi^{\circ}-\chi \\
f_{y}=y-y_{p} & f_{z}=z-z_{p} \\
\gamma=\arcsin \frac{\dot{z}_{g}}{V} & \chi=\arctan \frac{\dot{y}_{g}}{\dot{x}_{g}}
\end{array}
$$

and where $y_{p}$ is the current ceiling of the ATGM at a given moment of time; $y$ - programmed altitude of ATGM at a given moment of time; $z_{p}$ - factual location of ATGM; $z$ - programmed location of the ATGM; $\gamma, \chi$ - flight-path angle in the vertical and horizontal plane (inclination and azimuth angles of the missile velocity vector); $\dot{x}_{g}, \dot{y}_{g}, \dot{z}_{g}$ - components of the velocity vector in the ground-fixed system.

The indicated control signals $Q_{y}, Q_{z}$ for the purpose of simplification of the simulation can be considered as the controlling forces (Evans, 1990).

\section{Results of simulation tests}

Simulations are conducted for a hypothetical anti-tank guided missile whose mathematical model and equations of flight dynamics are presented in the previous Section. The data adopted for the missile are: $m=12.9 \mathrm{~kg}, e=0.45 \mathrm{~m}, l=0.231 \mathrm{~m}, I_{y}, I_{z}=1.53 \mathrm{~kg} \mathrm{~m}^{2}, I_{x}=0.0324 \mathrm{~kg} \mathrm{~m}^{2}$, $T_{R}=3700 \mathrm{~N}$ (launch motor), $T_{R}=400 \mathrm{~N}$ (flight motor).

\subsection{Simulation conducted for the ATGM flying through three points and attacking the} target moving with a velocity of $30 \mathrm{~m} / \mathrm{s}$

The autopilot regulator parameters are selected as follows: $k_{y 1}=400, k_{y 2}=320, k_{y 3}=10$, $h_{y 1}=900, h_{y 2}=36, h_{y 3}=2300, k_{z 1}=400, k_{z 2}=170, k_{z 3}=1000, h_{z 1}=900, h_{z 2}=8$, $h_{z 3}=1000$.

\subsection{Simulation conducted for the ATGM flying through four points and attacking the target moving with a velocity of $30 \mathrm{~m} / \mathrm{s}$}

The autopilot regulator parameters are selected as follows: $k_{y 1}=400, k_{y 2}=320, k_{y 3}=10$, $h_{y 1}=900, h_{y 2}=36, h_{y 3}=2300, k_{z 1}=200, k_{z 2}=250, k_{z 3}=1000, h_{z 1}=900, h_{z 2}=23$, $h_{z 3}=1000$. 


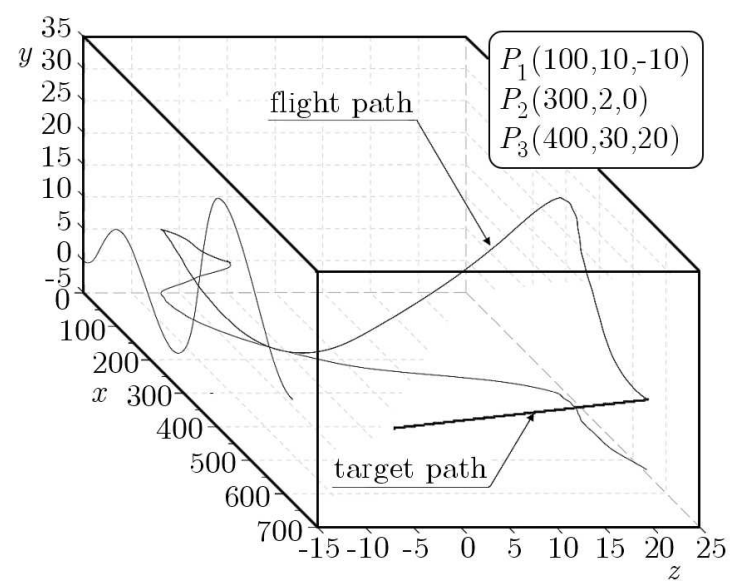

Fig. 7. Trajectory of the flight of the ATGM attacking the target moving with a velocity of $30 \mathrm{~m} / \mathrm{s}$.

The target moves 100 to the left and 50 up

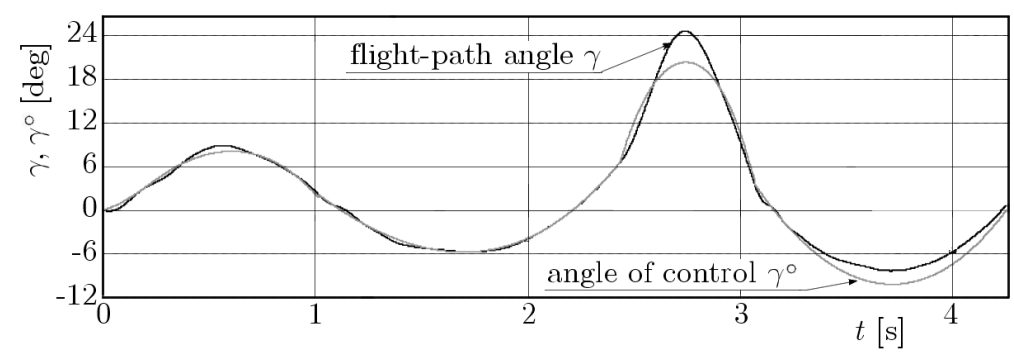

Fig. 8. The flight-path angle $\gamma$ and the control angle $\gamma^{\circ}$ in function of time

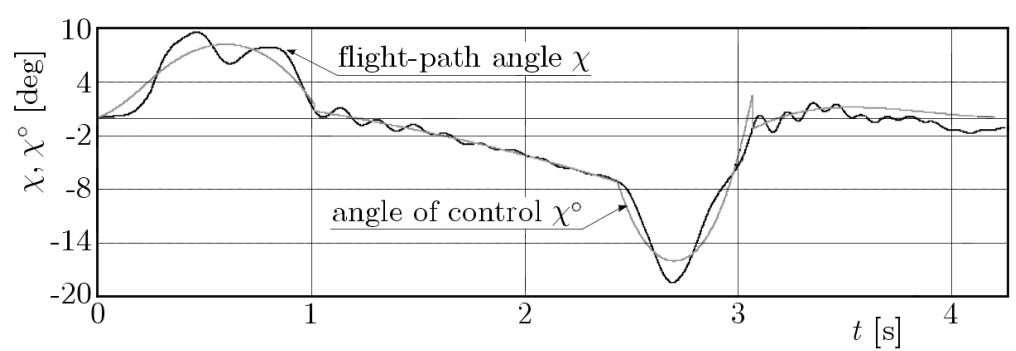

Fig. 9. The flight angle $\chi$ and the control angle $\chi^{\circ}$ in function of time

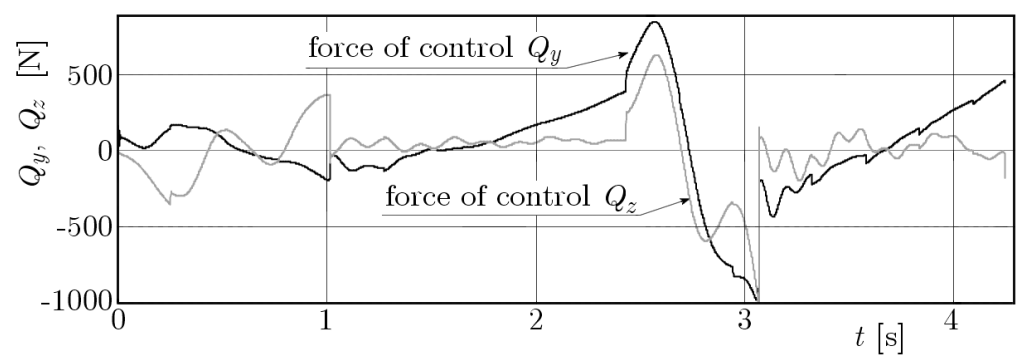

Fig. 10. Control signals of the ATGM: $Q_{y}$ controlling the altitude in the vertical plane, $Q_{z}$ controlling the direction in the horizontal plane 


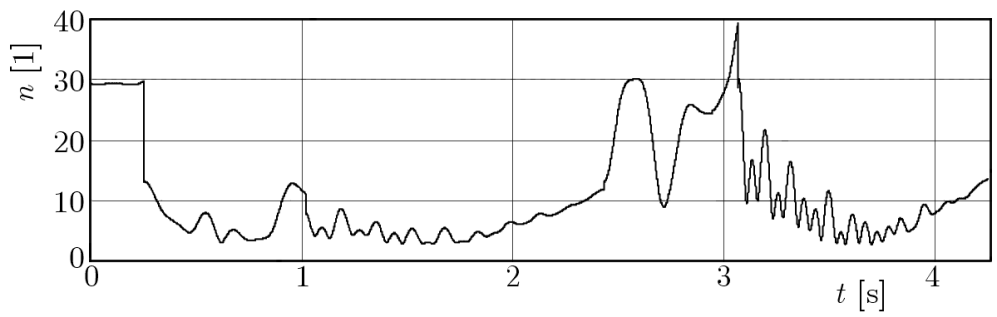

Fig. 11. Lateral overloads that affect the ATGM during the guidance

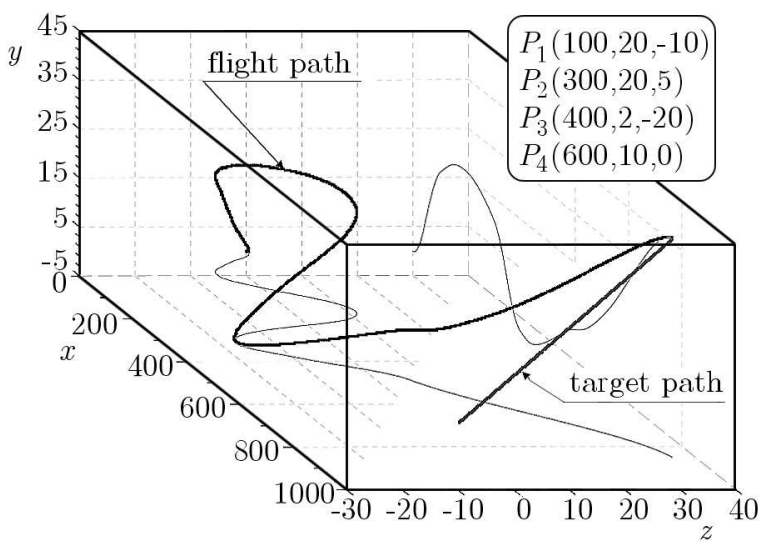

Fig. 12. Trajectory of the flight of the ATGM attacking the target moving with a velocity of $30 \mathrm{~m} / \mathrm{s}$. The target moves 150 to the left and 100 up

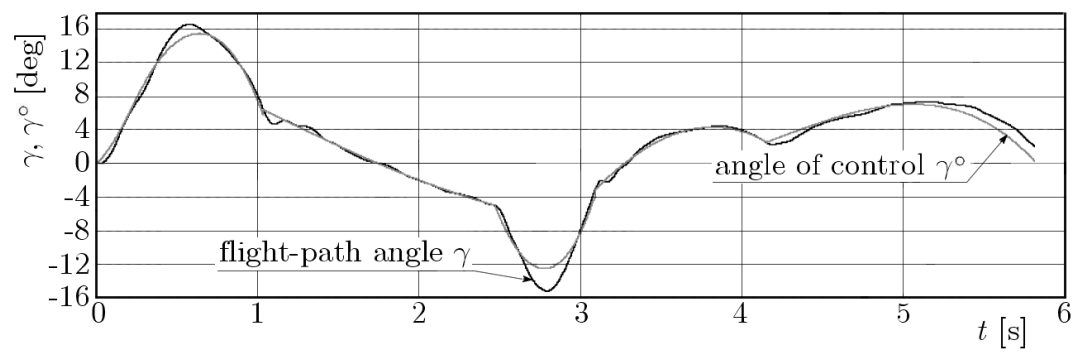

Fig. 13. The flight-path angle $\gamma$ and the control angle $\gamma^{\circ}$ in function of time

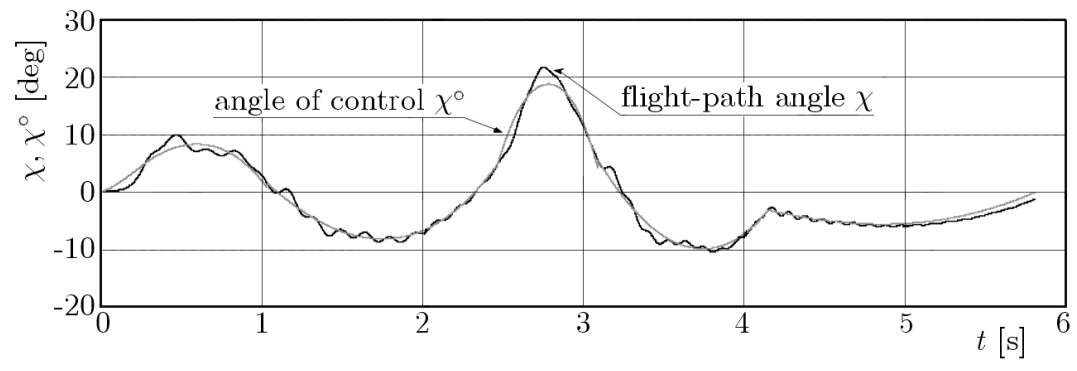

Fig. 14. The flight angle $\chi$ and the control angle $\chi^{\circ}$ in function of time 


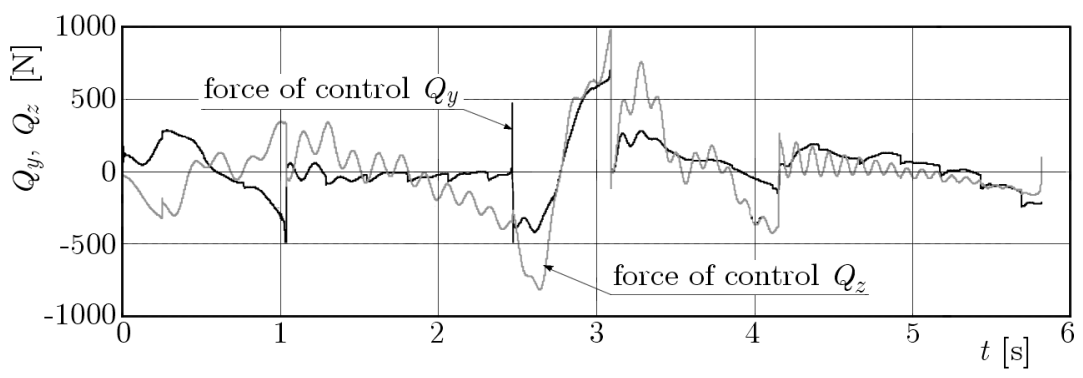

Fig. 15. Control signals of the ATGM: $Q_{y}$ controlling the altitude in the vertical plane, $Q_{z}$ controlling the direction in the horizontal plane

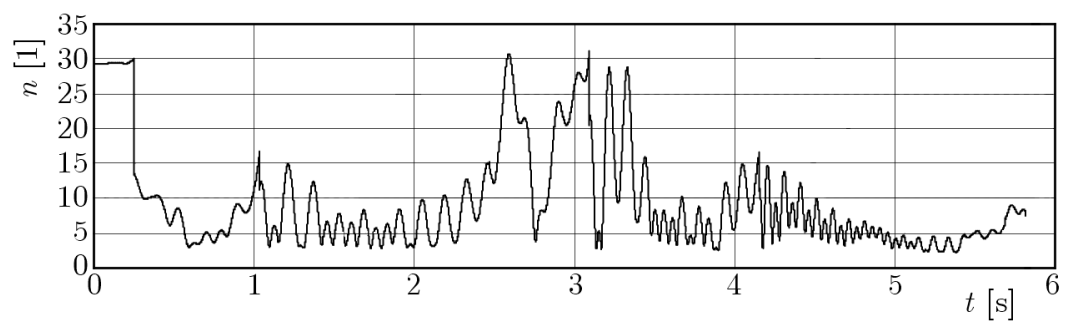

Fig. 16. Lateral overloads that affect the ATGM during the guidance

\section{Conclusions and final remarks}

From the conducted theoretical deliberations and simulation studies, one can draw the following conclusions:

- Proper selection of the regulator and its settings significantly affects not only the accuracy of mapping of the programmed trajectory, but also the optimal values of control signals as well as congestion during missile flight.

- Regulator settings are chosen in such a way so as to optimize the values of control signals and the existing congestions. It should be emphasized that the regulator gains assume values that are technically achievable. Admittedly, the angles of the missile flight do not coincide exactly with the control angles, and the trajectory does not perfectly map the trajectory of the program, however, it does not significantly affect the effectiveness of the attack.

- ATGM flies through designated points with a sufficient accuracy (one meter), and hits the target with a high accuracy (approx. half a meter). These results are satisfactory because of the fact that the target is moving at a relatively high velocity (about $30 \mathrm{~m} / \mathrm{s}$ ).

- In the points of gluing functions (subsequent parts of the trajectory), one can observe significant jumps of the control signals. This is due to the discontinuity of angular velocity functions in these points.

\section{References}

1. BARANOWski L., 2013, Equations of motion of spin-stabilized projectile for flight stability testing, Journal of Theoretical and Applied Mechanics, 51, 1, 235-246

2. Evans D.M., 1990, The direct-fire anti-armour capability of UK land forces, Intern, Defense Review, 7, 739-742 
3. Gapiński D., Stefański K., 2014, Modified optical scanning and tracking head for identification and tracking air targets, Solid State Phenomena, 210, 145-155, DOI: 10.4028/www.scientific.net/SSP.210.145

4. Grzyb M., Koruba Z., 2011, Guiding a bomb to a sea target (in Polish), Research Journal of Polish Naval Academy, 185A, ISSN 0860-889X, 189-195

5. Harris J., Slegers N., 2009, Performance of a fire-and-forget anti-tank missile with a damaged wing, Mathematical and Computer Modelling, 50, 1/2, 292-305

6. Koruba Z., 2008, The Elements of the Theory and Applications of the Controlled Gyroscope (in Polish), Monographs, Studies, Dissertations M7, Kielce University of Technology, Kielce

7. Koruba Z., Nocoń Ł., 2012, Selected algorithms of automatic guidance of anti-tank missiles attacking targets from upper ceiling (in Polish), Pomiary, Automatyka, Robotyka, 2

8. Koruba Z., Osiecki J., 2006, Structure, Dynamics and Navigation of Chosen Precision Kill Weapons, Publishing House of Kielce University of Technology, ISBN 83-88906-17-8, Kielce, 50-209

9. Krzysztofik I., 2012, The dynamics of the controlled observation and tracking head located on a moving vehicle, Solid State Phenomena, 180, 313-322, ISSN 1012-0394, DOI: 10.4028/www.scientific.net/SSP.180.313

10. Nocoń Ł., 2013, Detection control system of a missile safe distance trajectory, Proceedings of the 10th European Conference of Young Researchers and Scientists, EDIS - Żilina University Publisher, ISBN 978-80-554-0694-7, Transcom 2013, Żylina, 139-142

11. Nocoń Ł., Stefański K., 2014, The analysis of anti-tank rocket dynamics including external disturbances (in Polish), [In:] Mechanika w Lotnictwie, ML-XVI 2014, K. Sibilski (edit.), PTMTS Warsaw, ISBN 978-83-932107-3-2, 409-426

12. Siouris G.M., 2004, Missile Guidance and Control Systems, Springer, New York

13. Yanushevsky R., 2008, Modern Missile Guidance, CRC Press Taylor \& Francis Group, New York 